OPEN ACCESS

Method to Determine the In-Plane Tortuosity of Porous Electrodes

To cite this article: Bharatkumar Suthar et al 2018 J. Electrochem. Soc. 165 A2008

View the article online for updates and enhancements. 


\title{
Method to Determine the In-Plane Tortuosity of Porous Electrodes
}

\section{Bharatkumar Suthar, $\oplus^{\mathrm{z}}$ Johannes Landesfeind, ${ }^{*}$ Askin Eldiven, and Hubert A. Gasteiger**}

Chair of Technical Electrochemistry, Department of Chemistry and Catalysis Research Center, Technical University of Munich, Munich, Germany

\begin{abstract}
The tortuosity of a porous electrode is one of the critical parameters that governs the effective transport properties such as effective conductivity and effective diffusivity of ions in the electrolyte phase. While there exist several methods to determine the through-plane tortuosity for lithium-ion battery electrodes, methods to determine in-plane tortuosity are scarcely explored. In this paper, we present a method that restricts ion transport to only the in-plane direction, which facilitates the determination of transport properties in the in-plane direction. The here proposed cell design is analyzed using a blocking condition transmission line model to measure the in-plane tortuosity, followed by validation experiments to establish its validity and robustness. The measurement of the in-plane tortuosity may act as an indispensable tool for the design, characterization, and modeling of 3-dimensional battery architectures (e.g., laser structured electrodes) and help to estimate the distribution of various properties in the through-plane direction such as porosity, tortuosity, binder content, etc.

(C) The Author(s) 2018. Published by ECS. This is an open access article distributed under the terms of the Creative Commons Attribution Non-Commercial No Derivatives 4.0 License (CC BY-NC-ND, http://creativecommons.org/licenses/by-nc-nd/4.0/), which permits non-commercial reuse, distribution, and reproduction in any medium, provided the original work is not changed in any way and is properly cited. For permission for commercial reuse, please email: oa @electrochem.org. [DOI: 10.1149/2.0121810jes]
\end{abstract}

(cc) BY-NC-ND

Manuscript submitted April 26, 2018; revised manuscript received June 19, 2018. Published June 30, 2018.

Except for thin-film designs, lithium-ion batteries are composed of porous electrodes to mitigate the transport resistance of the relatively slow lithium diffusion in the solid phase of the active materials. Porous electrodes are composed of active material particles and conductive carbon additives held together by a suitable binder, with the resulting void space filled by an ion-conducting electrolyte. While this reduces the length scale for solid phase diffusion within the active material phase and provides a large interfacial area for the charge transfer reactions at the electrode/electrolyte interface, the tortuosity of the resulting ionic path within the electrolyte phase can vary substantially. The latter is affected by the shape and size distribution of the active material particles, by the type and amount of binder materials and conductive carbons, by the extent of electrode calendering (i.e., by the remaining electrolyte volume fraction), and by the specifics of the drying process during electrode preparation from electrode ink slurries; ${ }^{1-4}$ furthermore, ionic resistances through the electrode or parts of the electrode may increase over extended charge/discharge cycling of lithium-ion battery cells, effectively increasing electrode tortuosity. ${ }^{5}$

For traditional battery designs based on homogeneous nonstructured porous electrodes, the through-plane tortuosity is the most relevant parameter describing ion conduction in the electrolyte phase, as it is dominated by through-plane conduction. However, modeling and optimization of the more recently proposed three-dimensionally (3D) structured electrode designs requires the values of both the inplane and the through-plane tortuosity. Examples are interdigitated battery architectures ${ }^{6,7}$ and laser structured electrodes, ${ }^{8-11}$ where the transport of lithium ions is not restricted to the through-plane direction. The quantification of both in-plane and through-plane tortuosities of porous electrodes can also help in determining inhomogeneities in the through-plane direction produced, e.g., by the presence of a binder gradient ${ }^{12}$ or by lowering electrode porosity near the separator interface caused by continuous SEI (solid electrolyte interphase) formation during battery aging. ${ }^{5,13}$ The availability of experimental in-plane along with through-plane tortuosity values will help to tune and validate the numerical algorithms used to estimate the tortuosity from 3D reconstructions based on X-ray tomography and/or FIB-SEM analysis of battery electrodes. ${ }^{14-18}$

Experimentally, various methods have been applied to determine the tortuosity of battery electrodes. These methods often consist of either forcing ionic transport or gas transport through porous media

\footnotetext{
*Electrochemical Society Student Member.

**Electrochemical Society Fellow.

${ }^{\mathrm{z}}$ E-mail: bharat.k.suthar@tum.de
}

using a variety of experimental configurations ${ }^{1,19-21}$ and are usually focused on transport in the through-plane direction. Experimental determination of the in-plane tortuosity for battery electrodes and for fuel cell gas diffusion layers (GDLs) has also been demonstrated. ${ }^{14,22-25}$ For battery electrodes, Ebner et al. ${ }^{14}$ have used 3D representations of electrodes microstructures using X-ray tomographic microscopy (XTM) and performed numerical diffusion simulations to calculate the in-plane and through-plane tortuosities. The result of such numerical simulations heavily depend on the resolution of the 3D reconstructions in order to resolve the binder and conductive carbon. Inability to resolve the binder may lead to an underestimation of the tortuosity. ${ }^{26}$ High resolution FIBSEM reconstructions on the other hand suffer from long measurement times and resulting non-representative reconstructed volumes.

For fuel cell gas diffusion layer, Baker et al. ${ }^{22}$ assumed a constant ratio between in-plane and through-plane diffusion coefficients and then fit experimental data with detailed numerical simulations of the common geometry found in fuel cells (flow channel/land and diffusion layer). Mathias et al. ${ }^{23}$ used a similar approach as Baker et al. where they facilitated the gas transport between two flow channels through a diffusion layer. Rashapov et al. ${ }^{24}$ used an experimental setup that restrict the flow of oxygen in the in-plane direction and measured the concentration change at the center of the sheet. From the concentration evolution of oxygen, they calculated the in-plane tortuosity of the gas diffusion layer at various compressions. Kramer et al. ${ }^{25}$ has used electrochemical impedance based method with a specialized setup to measure the in-plane tortuosity. The method described above for the fuel cell gas diffusion layer either requires the use of detailed numerical simulations, or a specialized setup to measure the in-plane tortuosity, whereas work presented in this paper is simple enough to even work with a standard 2032 coin cell without detailed numerical simulations.

In this paper, we use electrochemical impedance spectroscopy (EIS) to extract the effective ionic transport resistance in a porous medium. ${ }^{27}$ For lithium-ion battery electrodes, the use of electrochemical impedance spectroscopy (EIS) based on a transmission line model (TLM) analysis to extract the effective through-plane transport resistance in a porous electrode under both non-blocking and blocking conditions (where faradaic reactions are suppressed) was shown by Ogihara et al. ${ }^{20}$ Landesfeind et al. ${ }^{1}$ presented a setup to measure the through-plane tortuosity of various electrode materials as a function of porosity and electrode composition using a non-intercalating electrolyte and thereby forcing blocking conditions.

Here, we use a novel measurement configuration which, for the first time, enables the quantification of the in-plane tortuosity 


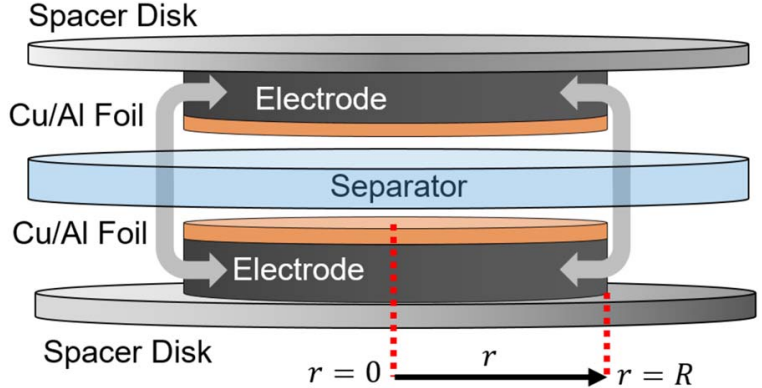

Figure 1. Sketch of the experimental setup to measure the in-plane tortuosity of porous electrodes. The solid gray arrows indicate in-plane transport of ions (sketch not drawn to scale). Here, $r$ is the radial coordinate, which goes from the center of the disk $(r=0)$ to the perimeter of the disk $(r=R)$.

of porous lithium-ion battery electrodes by EIS conducted with a non-intercalating electrolyte ("blocking electrolyte"). First, we present the conceptual design of the experimental setup, the governing equations for a TLM analysis for the given geometry, and its analytical solution which provides the effective in-plane ionic resistance in the electrolyte phase. Then we discuss the analytical solution in detail and establish the validity of the proposed method by conducting coin cell experiments for different experimental design parameters: i) electrode thickness and diameter; ii) bulk conductivity of the electrolyte; and, iii) electrode alignment requirements. We will show, that our rather simple experimental setup and data analysis yields reproducible and reliable in-plane tortuosity values and that the obtained Nyquist plots are consistent with our analytical solution.

\section{Theory}

Figure 1 shows a conceptual design of a coin cell setup that restricts the transport of ions in the electrolyte phase to the in-plane direction. Contrary to the symmetric cell design for the through-plane tortuosity determination where the electrodes coated on a current collector foil and separated by a porous separator face each other, ${ }^{1,20}$ in this case the electrodes are flipped so that the current collectors face the separator (see Figure 1). In this configuration, the ionic transport in the electrolyte phase within the electrodes is restricted in the through-plane direction by the current collector on one side and the cell (here the spacer disk) on the other side. Note that the various parts shown in Figure 1 are not drawn to scale. Usually, the battery electrode thickness would range from 50-200 $\mu \mathrm{m}$, whereas the diameter of the electrode disks can be as large as $\sim 15 \mathrm{~mm}$ for 2032-type coin cells.

Since the cell design as shown in Figure 1 has axial symmetry, we analyze the governing equations for a disk in cylindrical coordinates where the transport is restricted only to the radial (in-plane) direction. The ionic flux in a porous media - in the context of the here applied TLM, only migration must be considered - is given by $-\kappa_{\text {eff }} \nabla \phi$, where $\kappa_{\text {eff }}$ is the effective conductivity of ions in the electrolyte phase, and $\phi$ is the electrolyte potential. ${ }^{28}$ Here, $\kappa_{\text {eff }}$ is defined as

$$
\kappa_{\mathrm{eff}} \equiv \kappa \frac{\varepsilon}{\tau}
$$

where $\varepsilon$ is the porosity of the electrode, $\tau$ is the tortuosity of the electrode, and $\kappa$ is the bulk ionic conductivity of electrolyte. In this work, the effective conductivity is assumed to be constant, both temporally and spatially. In an infinitesimally small control volume, the general charge conservation equation for the non-intercalating electrolyte (i.e., no faradaic reaction) incorporating ionic flux and the double layer capacitance can be written as follows.

$$
a C_{\mathrm{dl}} \frac{\mathrm{d}}{\mathrm{d} t} \phi=-\nabla \cdot\left(-\kappa_{\mathrm{eff}} \nabla \phi\right)
$$

where $a\left[\mathrm{~m}^{2} / \mathrm{m}^{3}\right]$ is the specific surface area per electrode volume of both the active material and the conductive carbon, $C_{\mathrm{dl}}\left[\mathrm{F} / \mathrm{m}^{2}\right]$ is the areal double layer capacitance, and $t$ is the time. The specific surface area per electrode volume $(a)$ can be obtained experimentally from the BET measurements, the electrode loadings, and the electrode thickness. The reason for ignoring diffusion flux in the setup is because the intercalation/de-intercalation reaction is blocked and only capacitance effects are invoked, hence no concentration gradients can evolve. At every point of the cell throughout the measurement, the local ion concentration remains constant. For the disk geometry (cylindrical coordinates) shown in Figure 1, which is axis-symmetric, Eq. 2 can be simplified as

$$
a C_{\mathrm{dl}} \frac{\mathrm{d}}{\mathrm{d} t} \phi=-\frac{1}{r} \frac{\mathrm{d}}{\mathrm{d} r} r\left(-\kappa_{\mathrm{eff}} \frac{\mathrm{d}}{\mathrm{d} r} \phi\right)
$$

where $r$ denotes the radial coordinate which goes from the center of the disk $(r=0)$ to the perimeter of the disk $(r=R)$. We convert Eq. 3 into the frequency domain.

$$
a C_{\mathrm{dl}} i w \boldsymbol{\Phi}=-\frac{1}{r} \frac{\mathrm{d}}{\mathrm{d} r} r\left(-\kappa_{\mathrm{eff}} \frac{\mathrm{d}}{\mathrm{d} r} \boldsymbol{\Phi}\right)
$$

where $\boldsymbol{\Phi}$ is the complex potential, $w$ is the angular frequency, and $i$ is the imaginary unit. The no-flux boundary condition at the center of the disk and an arbitrarily set value for the complex potential at the outer perimeter of the disk are given by Eqs. 5 and 6 .

$$
\begin{gathered}
-\left.\kappa_{\mathrm{eff}} \frac{\mathrm{d}}{\mathrm{d} r} \boldsymbol{\Phi}\right|_{r=0}=0 \\
\left.\boldsymbol{\Phi}\right|_{r=R}=1
\end{gathered}
$$

The complex impedance at $r=R$ is defined as the ratio of complex potential ( = 1, see Eq. 6) to the magnitude of the complex current for the given disk of radius $R$ and thickness $d$ as follows. ${ }^{29}$

$$
\mathbf{Z}=\left.\frac{\boldsymbol{\Phi}}{(2 \pi R d) \kappa_{\mathrm{eff}} \frac{\mathrm{d} \boldsymbol{\Phi}}{\mathrm{d} r}}\right|_{r=R}
$$

Eqs. 4, 5, and 6 describe the governing system of equations that needs to be solved in order to get the complex impedance $\mathbf{Z}$ of a single disk (Eq. 7) for which the transport is restricted only in the radial direction. We define the dimensionless radial distance $\xi=r / R$ and the characteristic angular frequency $w_{\mathrm{c}}=\kappa_{\mathrm{eff}} /\left(a C_{\mathrm{dl}} R^{2}\right)$, based on which we rewrite Eqs. 4, 5, 6, and 7 into the following system of equations.

$$
\begin{gathered}
\frac{i w}{w_{\mathrm{c}}} \boldsymbol{\Phi}=-\frac{1}{\xi} \frac{\mathrm{d}}{\mathrm{d} \xi} \xi\left(-\frac{\mathrm{d}}{\mathrm{d} \xi} \boldsymbol{\Phi}\right) \\
\left.\frac{\mathrm{d} \boldsymbol{\Phi}}{\mathrm{d} \xi}\right|_{\xi=0}=0 \\
\left.\boldsymbol{\Phi}\right|_{\xi=1}=1 \\
\mathbf{Z}=\left.\frac{\boldsymbol{\Phi}}{(2 \pi d) \kappa_{\mathrm{eff}} \frac{\mathrm{d} \boldsymbol{\Phi}}{\mathrm{d} \xi}}\right|_{\xi=1}
\end{gathered}
$$

Solution of the second order differential equation with the associated boundary conditions given in Eqs. 8-10 can be found as a linear combination of Bessel functions of the first $\left(J_{n}\right)$ and the second kind $\left(Y_{n}\right)$ of order $n$.

$$
\boldsymbol{\Phi}=c_{1} J_{0}(S \xi)+c_{2} Y_{0}(S \xi)
$$

Here, $c_{1}$ and $c_{2}$ are constants, $J_{0}$ and $Y_{0}$ are Bessel functions of the first and the second kind of order 0 and $S \equiv \sqrt{-i w / w_{\mathrm{c}}}$. Boundary conditions at the center $(\xi=0)$ and at the perimeter $(\xi=1)$ of the electrode disk can be applied to obtain the value of constants $c_{1}$ and $c_{2}$. At the center of the disk electrode $(\xi=0)$ the value of $Y_{0}(x \rightarrow 0) \rightarrow-\infty$, so that in order for $\boldsymbol{\Phi}$ to be finite at the center 
of the disk, $c_{2}$ will have to be zero. Applying the boundary condition $\left.\boldsymbol{\Phi}\right|_{\xi=1}=1$, we get the following equation for the complex potential.

$$
\boldsymbol{\Phi}=\frac{J_{0}(S \xi)}{J_{0}(S)}
$$

The complex impedance at $\xi=1$ (i.e., at $r=R$ ) is given by substituting Eq. 13 into Eq. 11, which yields

$$
\mathbf{Z}=-\frac{1}{2 \pi d \kappa_{\mathrm{eff}}} \frac{J_{0}(S)}{S J_{1}(S)}
$$

Eq. 14 represents the solution of the impedance of a single electrode disk in the radial direction. Since in the experimental setup, two nominally identical disks are connected in series with each other, the impedance of the two nominally identical disks $\left(\mathbf{Z}_{\text {sys }}\right)$ is given as

$$
\mathbf{Z}_{\mathrm{sys}}=-\frac{1}{\pi d \kappa_{\mathrm{eff}}} \frac{J_{0}(S)}{S J_{1}(S)}
$$

In order to get the intercept of the impedance curve at the real axis as $w \rightarrow 0$, the series expansion of Eq. 15 with respect to $w$ is used, and $w=0$ is substituted. Using this approach, we get the following value for the low-frequency intercept $L$ for the two-electrode system described in Figure 1.

$$
L=\frac{1}{4 \pi d \kappa_{\mathrm{eff}}}
$$

It is noteworthy that $L$ is independent of the radius of the electrode disks, meaning the setup described in Figure 1 should lead to the same value of the low-frequency intercept in a Nyquist plot, irrespective of the diameter of the disks. This useful property will be used later on to validate this method experimentally. The effect of electrode thickness and electrolyte conductivity on the ionic resistance is also very clear from Eq. 16: doubling the electrode thickness or electrolyte conductivity will reduce the ionic resistance by a factor of two. It should be noted that the above analysis is valid for a case with an ideal capacitance. Practical electrodes behave more like distributed constant phase elements rather than ideal distributed capacitors, hence, for actual experimental data, the linear region of the low-frequency impedance curve is used to obtain the value of the low-frequency intercept by extrapolation. Finally, using Eq. 1, Eq. 16 can be rewritten in the following form, which will be used in the Results section to extract the in-plane tortuosity of a porous electrode from the measured low-frequency intercept.

$$
\tau_{\mathrm{i}-\mathrm{p}}=4 \pi d \kappa \varepsilon L
$$

To simulate the impedance response for a coin cell setup with two nominally identical battery electrodes (see Figure 1) using Eq. 15, the following representative physical-chemical parameter values were chosen: i) a porosity of $\varepsilon=0.5$, ii) an in-plane tortuosity of $\tau_{\mathrm{i}-\mathrm{p}}=2$, iii) a bulk electrolyte ionic conductivity of $\kappa=5$ $\mathrm{mS} / \mathrm{cm}$, iv) a thickness $d=100 \mu \mathrm{m}$, and, v) a volumetric capacitance of $a C_{\mathrm{dl}}=20,000 \mathrm{~F} / \mathrm{m}^{3}$ (specific surface area $a$ from a BET surface area of $\sim 0.2 \mathrm{~m}^{2} / \mathrm{g}$ (e.g. for NMC, for graphite $\sim 2 \mathrm{~m}^{2} / \mathrm{g}$ ) and an electrode loading of $10 \mathrm{mg} / \mathrm{cm}^{2}$ and an electrode thickness of $100 \mu \mathrm{m}$ and an areal double layer capacitance of $\left.\sim 10 \mu \mathrm{F} / \mathrm{cm}^{2}\right) .{ }^{30}$ This would correspond to an effective conductivity of $\kappa_{\text {eff }}=1.25 \mathrm{mS} / \mathrm{cm}$ (see Eq. 1) and for three different sets of disk radii $(2.5,5$, and $7.5 \mathrm{~mm})$, the characteristic angular frequencies $\left(w_{\mathrm{c}}=\right.$ $\left.\kappa_{\mathrm{eff}} /\left(a C_{\mathrm{dl}} R^{2}\right)\right)$ would equate to $w_{c}=1,1 / 4$, and $1 / 9 \mathrm{rad} / \mathrm{s}$, respectively (corresponding to characteristic frequencies of $f_{\mathrm{c}}=160,40$, and $18 \mathrm{mHz}$ ); on the other hand, the low-frequency intercept would have the identical value of $L=6.366 \mathrm{k} \Omega$ for these three electrodes (see Eq. 16). The Nyquist and the Bode plot representations of Eq. 15 for the three different disk sizes simulated for the electrode configuration given in Figure 1 are shown in Figure 2. As expected, the curves for the three differently sized electrodes look identical in the Nyquist plot but differ in the Bode plot.

We also define the turning frequency $\left(f_{\mathrm{t}}\right)$ as the point where the imaginary part of the impedance is equal to the low-frequency intercept $L\left(\operatorname{Im}\left(\mathbf{Z}_{\text {sys }}\left(f_{\mathrm{t}}\right)\right)=L\right)$. The ratio of turning frequency to the
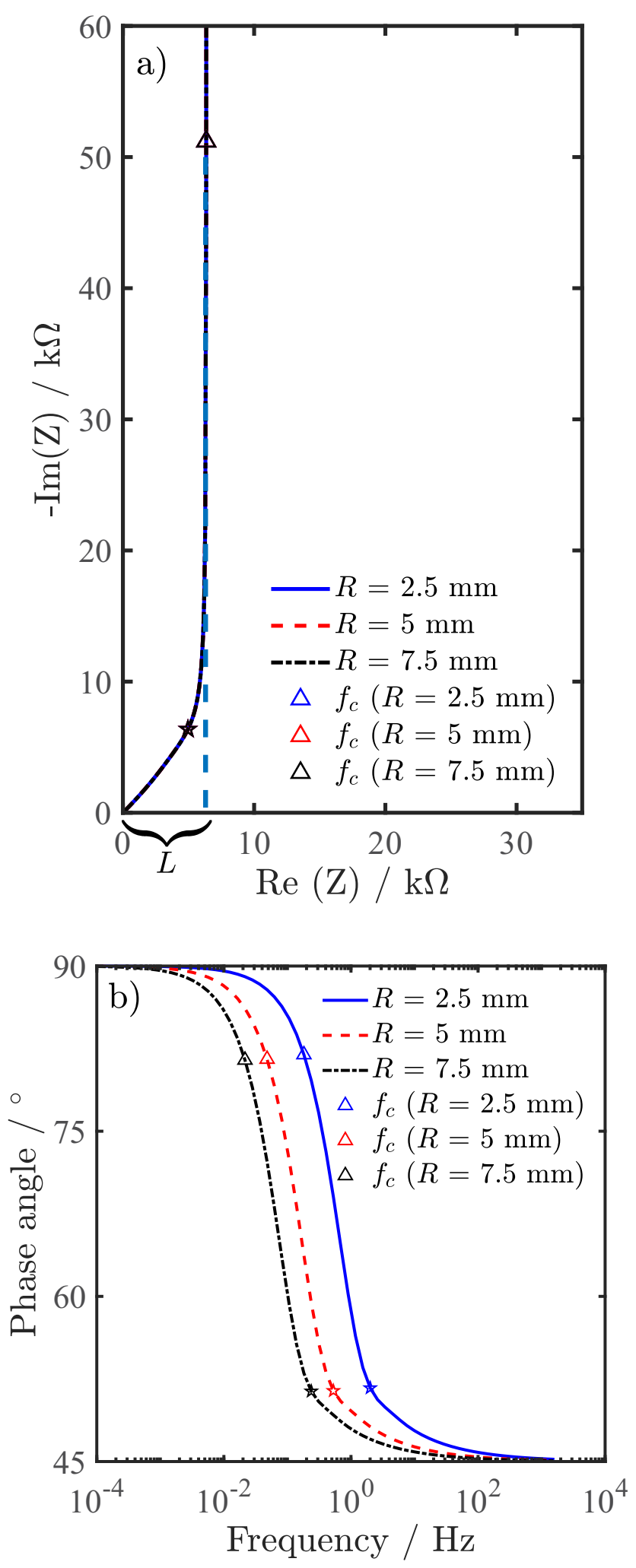

Figure 2. Simulated Nyquist (a) and Bode (b) plots for the two-electrode configuration shown in Figure 1 for three different disk radii $(R=2.5,5$, and $7.5 \mathrm{~mm}$ ). Although the low-frequency intercept $L$ is identical for all three disk sizes (see Eq. 16), the frequency at which the transition from near $45^{\circ}$ to $90^{\circ}$ angle occurs depends on the disk radius. Model electrode parameters: i) a porosity of $\varepsilon=0.5$, ii) an in-plane tortuosity of $\tau_{\mathrm{i}-\mathrm{p}}=2$, iii) a bulk electrolyte ionic conductivity of $\kappa=5 \mathrm{mS} / \mathrm{cm}$, and, iv) a volumetric capacitance of $a C_{\mathrm{dl}}=20,000 \mathrm{~F} / \mathrm{m}^{3}$. The characteristic frequencies $f_{\mathrm{c}}\left(f_{\mathrm{c}}=\right.$ $\left.\kappa_{\mathrm{eff}} /\left(2 \pi a C_{\mathrm{dl}} R^{2}\right)\right)$ are marked by the triangular symbols. The turning frequencies $f_{t}\left(f_{t} \approx 11.71 f_{c}\right)$ are marked with a star symbol. 


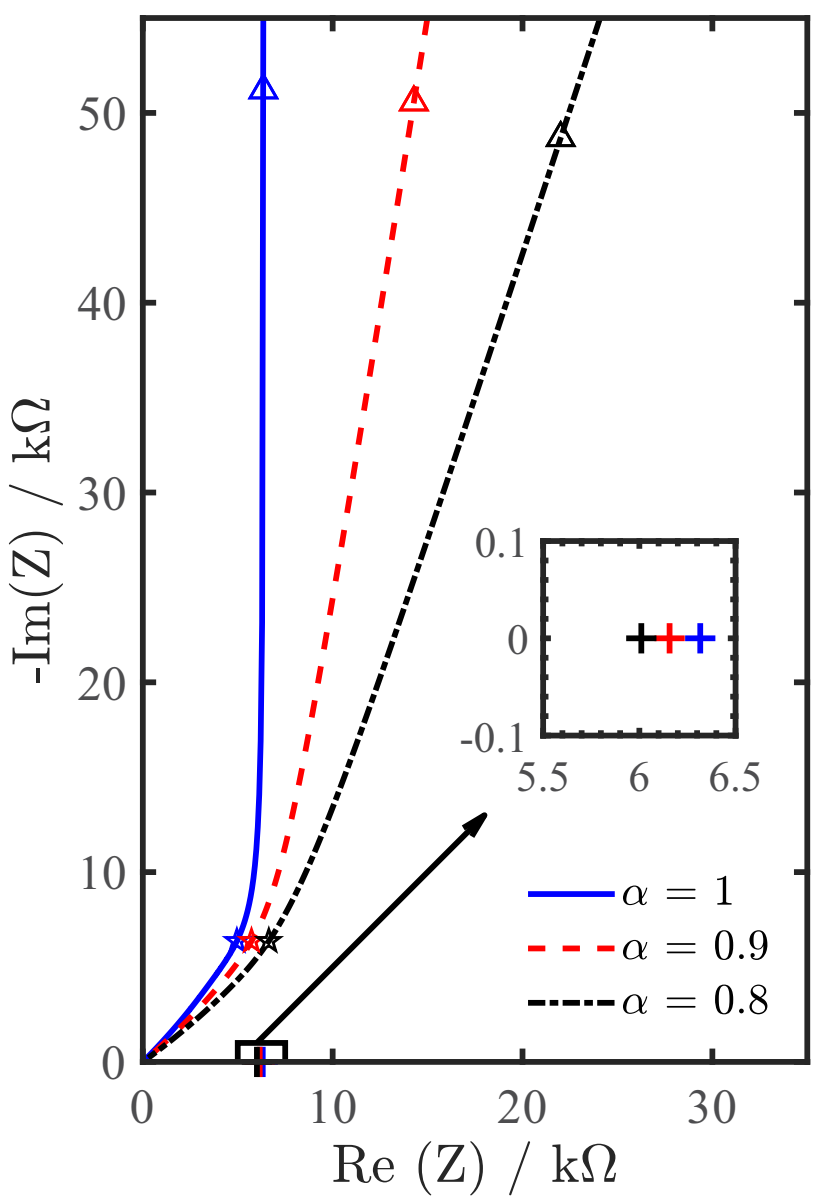

Figure 3. Simulated Nyquist plots for the two-electrode configuration shown in Figure 1 for a disk radius of $5 \mathrm{~mm}$ and three different values of the constant phase parameter $\left(\alpha=1,0.9\right.$, and 0.8). The characteristic frequencies $f_{\mathrm{c}}\left(f_{\mathrm{c}}=\right.$ $\left.\kappa_{\mathrm{eff}} /\left(2 \pi a C_{\mathrm{dl}} R^{2}\right) \approx 40 \mathrm{mHz}\right)$ are marked by the triangular symbols. Linear extrapolation using the points in the immediate vicinity of the characteristic frequency leads to the intercept on the $x$-axis (marked by the + symbols, also shown in the inset). These intercepts $\left(L_{\text {ext }}\right)$ underpredict the true value of $L$ by $\sim 1 \%, \sim 3 \%$ and $\sim 6 \%$ for $\alpha=1,0.9$, and 0.8 respectively. The turning frequencies are marked by star symbols $\left(f_{\mathrm{t}}=0.47,0.56,0.69 \mathrm{~Hz}\right.$ for $\alpha=1$, 0.9 , and 0.8 ). The model electrode parameters are: i) a porosity of $\varepsilon=0.5$, ii) an in-plane tortuosity of $\tau_{\mathrm{i}-\mathrm{p}}=2$, iii) a bulk electrolyte ionic conductivity of $\kappa=5 \mathrm{mS} / \mathrm{cm}$, and, iv) a volumetric capacitance of $a C_{\mathrm{dl}}=20,000 \mathrm{~F} / \mathrm{m}^{3}$.

characteristic frequency is found to be 11.71 for the ideal distributed capacitance case. The turning frequency is marked with a star symbol in Figure 2. Note that this ratio $\left(f_{\mathrm{t}} / f_{\mathrm{c}}\right)$ is independent of the design parameters (e.g., $R, d, \kappa)$. The turning frequency can be used to estimate the frequency range necessary for the determination of the low frequency intercept with low error. The characteristic frequency (or the turning frequency for that matter) can be understood in terms of penetration depth. Starting from the high frequencies where the signal only probe around the periphery of the electrode $(r=R)$, as the frequency goes down and reaches the characteristic frequency, the signal begins to probe the whole of the electrode or starts to reach the end of the porous media (in this case the center of the electrode, or $r=0$ ).

Non-ideal capacitance.-As mentioned before the porous electrode behaves more like a distributed constant phase element than a distributed ideal capacitor. The solution given in Eq. 15 can be modified to take care of the constant phase element by redefining $S$ using a constant phase parameter $\alpha$ as $S_{\alpha}\left(\right.$ where $\left.S_{\alpha} \equiv \sqrt{-\left(i w / w_{c}\right)^{\alpha}}\right)$. The value of $\alpha$ is 1 for an ideal capacitance. Eq. 15 can be rewritten in

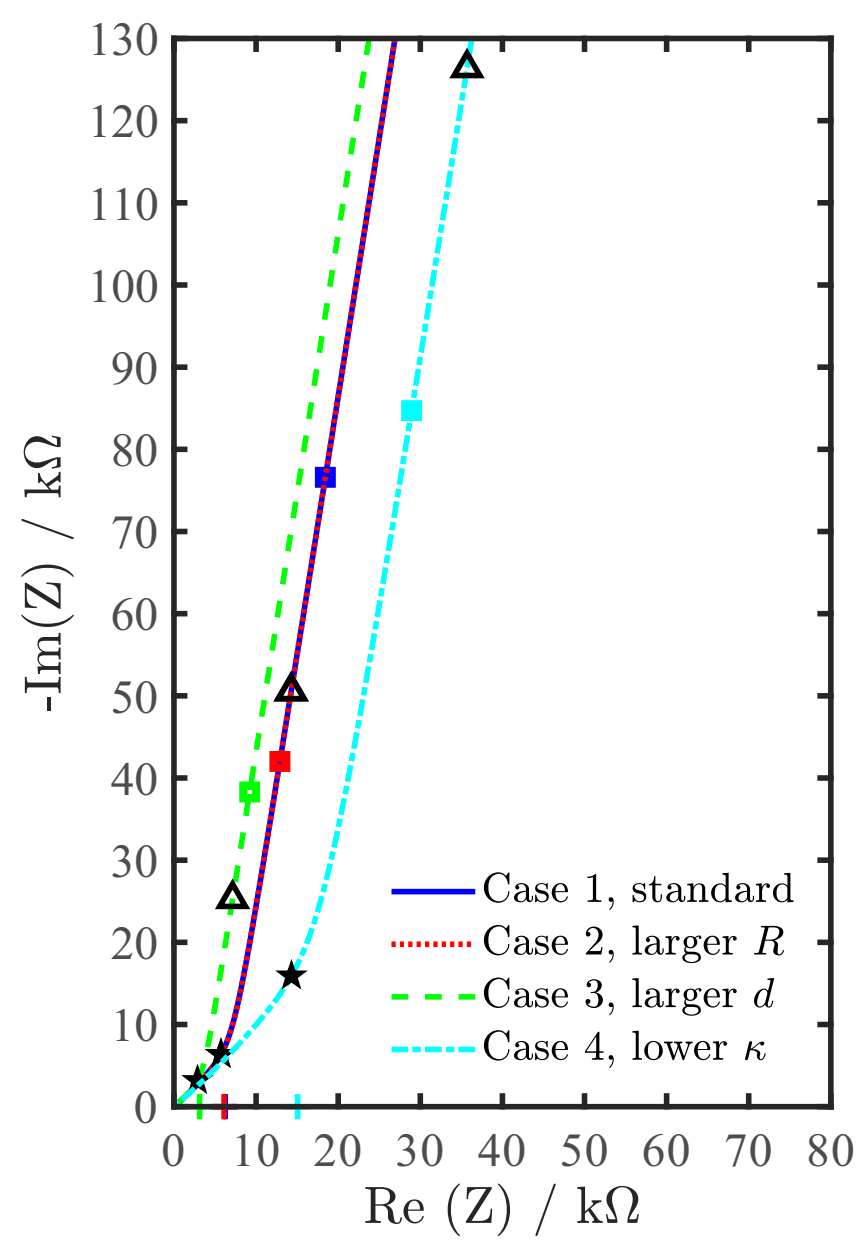

Figure 4. Simulated Nyquist plots for four different cases where one of the three parameters $(R, \kappa$, and $d)$ is varied. Case 1 is the base case with a radius of $R=5 \mathrm{~mm}$, a thickness of $d=100 \mu \mathrm{m}$, and a conductivity of $\kappa=5 \mathrm{mS} / \mathrm{cm}$. Case 2 has a larger diameter (radius $7 \mathrm{~mm}$ ), Case 3 has double the thickness, and Case 4 has a lower conductivity $(2 \mathrm{mS} / \mathrm{cm})$ compared to bases case (i.e., Case 1$)$. The characteristic frequencies $f_{\mathrm{c}}\left(f_{\mathrm{c}} \approx 40,20,40\right.$, and $20 \mathrm{mHz}$ for case 1 to case 4 respectively) are marked by the triangular symbols. Note that for Case 1 and Case 2, the value of the impedance at the respective characteristic frequencies are the same, yet the value of $f_{\mathrm{c}}$ differs (see Table I). The extrapolation frequency $\left(f_{\min }=25 \mathrm{mHz}\right)$ is marked by the square symbol and is the same for all cases. The low-frequency intercepts derived from the linear extrapolation near $f_{\min }\left(L_{\text {ext }}\right)$ are marked with a ' + ' symbol. Underprediction for the theoretical value of $L$ is larger in the case when the characteristic frequency is lower than the extrapolation frequency (i.e., case 2 and case 4 ), which is summarized in Table I. The turning frequencies for each case are marked with star symbols (for $\alpha=0.9, f_{t} \approx 14.06 f_{c}$ ).

terms $S_{\alpha}$ as follows (Lasia 2014, Chapter 4): $:^{31}$

$$
\mathbf{Z}_{\mathrm{sys}, \alpha}=-\frac{1}{\pi d \kappa_{\mathrm{eff}}} \frac{J_{0}\left(S_{\alpha}\right)}{S_{\alpha} J_{1}\left(S_{\alpha}\right)}
$$

Keeping the values of all the parameters same as shown in Figure 2, we choose a disk radius of $5 \mathrm{~mm}$ and three different values of $\alpha(\alpha=1,0.9$, and 0.8$)$ and plot the calculated results of Eq. 18 in Figure 3. Figure 3 also shows the impedance values at the characteristic frequency for each $\alpha$. From the seemingly linear portion of the Nyquist plots in the vicinity of the characteristic frequency, the linear extrapolation results in 3 different values of the low-frequency intercept $\left(L_{\text {ext }}\right)$. All the three values of $L_{\text {ext }}$ underpredict the theoretical value of $L\left(=1 /\left(4 \pi d \kappa_{\text {eff }}\right)=6.366 \mathrm{k} \Omega\right)$ where smaller $\alpha$ values lead to a larger underprediction. The three values of $\alpha=1,0.9$, and 0.8 underpredict the $L$ value by $\sim 1 \%, \sim 3 \%$ and $\sim 6 \%$ respectively. Using a frequency range lower than the characteristic frequency will reduce 
Table I. Summary of four cases with different design parameters (see Figure 4), their corresponding characteristic frequencies, the theoretical value of the low-frequency intercept and the intercept value obtained using a linear extrapolation of the impedance values around the extrapolation frequency $f_{\min }=25 \mathrm{mHz}$. All intercept values $L_{\text {ext }}$ obtained by the extrapolation underpredict the theoretical value of the low-frequency intercept. The underprediction increases when the characteristic frequency becomes lower than the chosen extrapolation region (i.e. when $f_{\min }>f_{\mathrm{c}}$ ). For all the four cases with $\alpha=0.9$, the turning frequency $f_{t} \approx 14.06 f_{c}$.

\begin{tabular}{cccccccc} 
Cases & $R(\mathrm{~mm})$ & $\kappa(\mathrm{mS} / \mathrm{cm})$ & $d(\mu \mathrm{m})$ & $f_{\mathrm{c}}(\mathrm{mHz})$ & $L(\mathbf{k} \boldsymbol{\Omega})$ & $L_{\text {ext }}(\mathbf{k} \boldsymbol{\Omega})$ & Under Prediction \\
\hline Case 1, standard & 5 & 5 & 100 & 40 & 6.366 & 6.238 & $-2.0 \%$ \\
Case 2, larger $R$ & $\mathbf{7}$ & 5 & 100 & $\sim 20$ & 6.366 & 6.104 & $-4.1 \%$ \\
Case 3, larger $d$ & 5 & 5 & $\mathbf{2 0 0}$ & 40 & 3.183 & $-2.0 \%$ \\
Case 4, lower $\mathrm{k}$ & 5 & $\mathbf{2}$ & 100 & $\sim 16$ & 15.915 & 15.056
\end{tabular}

the underprediction of $L$. Hence lower frequencies than the characteristic frequency should be used to minimize this effect, but in most practical applications, the impedance at lower frequencies $(<1 \mathrm{mHz})$ are usually corrupted by temperature fluctuations, side reactions, etc. and require long testing times. The turning frequency can also be defined for cases with a distributed constant phase element in the same way as defined previously. The turning frequencies for different $\alpha$ values are plotted in Figure 3 (marked by the star symbols). Note that the ratios of turning frequency $\left(\operatorname{Im}\left(\mathbf{Z}_{\text {sys }}\left(f_{t}\right)\right)=L\right)$ to characteristic frequency $\left(f_{\mathrm{c}}=\kappa_{\mathrm{eff}} /\left(2 \pi a C_{\mathrm{dl}} R^{2}\right)\right)$ for $\alpha=1,0.9$, and 0.8 are $\sim 11.71$, 14.06 , and 17.34. For a given value of $\alpha$, the ratio remains constant irrespective of the cell design parameters (e.g., $R, \kappa_{\text {eff }}, d$, etc.).

In order to gain more understanding with regards to how the accuracy of extrapolated low-frequency intercept gets affected by the choice of design parameters (namely disk radius $R$, electrolyte conductivity $\kappa$, and electrode thickness $d$ ), we consider four different cases (plotted in Figure 4). Table I lists the four different cases where one of the parameters $(R, \kappa$, and $d)$ differs in each case. Each of the four cases has a porosity of $\varepsilon=0.5$, an in-plane tortuosity of $\tau_{i-p}=2$, and a volumetric capacitance of $a C_{\mathrm{dl}}=20,000 \mathrm{~F} / \mathrm{m}^{3}$. The characteristic frequencies for each case are marked by the triangular symbols. Note that for case 1 and case 2 (different diameters) the characteristic frequencies are different $(40 \mathrm{mHz}$ and $20 \mathrm{mHz}$ respectively), yet the impedance plotted in Figure 4 is identical.

To illustrate the point that using a fixed-cut off frequency for extrapolation purpose may lead to an error that is different in different cases, we choose $25 \mathrm{mHz}$ as a cutoff frequency for all four cases. The extrapolation frequency $\left(f_{\min }=25 \mathrm{mHz}\right)$ is marked with square symbols in Figure 4. Note that the extrapolation frequency (which is the same frequency for all four cases) is smaller than the characteristic frequency in two cases (standard and larger $d$ ), and larger in the other two cases (larger $R$ and lower $\kappa$ ). The extrapolation performed with the frequency range lower than the characteristic frequency will lead to smaller difference between $L_{\text {ext }}$ and $L$, i.e., a lower underprediction.

The simulation of Eq. 18 (see Figure 4 and Table I) sheds light on the choice of design parameters and their effect on the extrapolated low-frequency intercepts. The access to the lower frequency range (compared to the characteristic frequency) when the low-frequency data is not corrupted by fluctuations of e.g., the temperature of the climate-chamber or, by side reactions, is desirable to avoid the extrapolation related underprediction of the ionic resistance and thus the in-plane tortuosity of the sample. By tuning the design parameters, the characteristic frequency can be increased, hence improving the accuracy of the low-frequency intercept for the same extrapolation frequency. For a given electrode sheet, the characteristic frequency can be increased by i) using an electrolyte with a higher conductivity and ii) using a smaller disk radius. It is worth mentioning here that the disk radius cannot be reduced arbitrarily as the analysis is valid only for the case when the transport is restricted predominantly to the in-plane direction by choosing the radius of the disk significantly higher than the thickness of the electrode $(R \gg d)$.

The concept of turning frequency can be effectively utilized to make sure that the extrapolation related underprediction remains within an acceptable error range. From the experimentally obtained Nyquist plot, the extrapolation from the seemingly linear portion will give rise to a low frequency intercept $\left(L_{\mathrm{ext}}\right)$, A rough estimation of the turning frequency can be made by reading the Nyquist plot for a frequency where the imaginary part of impedance curve is equal to $L_{\mathrm{ext}}$. From this approximate turning frequency, an approximate characteristic frequency can be obtained $\left(f_{\mathrm{c}}\right.$ is at least an order of magnitude lower than $f_{\mathrm{t}}$, see Figure 3). Finally, choosing the minimum frequency $\left(f_{\min }\right)$ during the experiment lower than the characteristic frequency will make sure that the underprediction remains within a reasonable range.

\section{Experimental Setup}

Preparation of electrodes and electrolyte.-The electrode was prepared by doctor-blade coating a slurry of $97 \%$ wt meso carbon micro beads (MCMB, MTI CORP., $2.0 \mathrm{~m}^{2} / \mathrm{g}$ BET area, D50 $18 \mu \mathrm{m}$ ), 3\% $\%_{\mathrm{wt}}$ PVDF binder (Kureha, KF1100, with a density of $1.77 \mathrm{~g} / \mathrm{cm}^{3}$ ) and NMP (Sigma Aldrich, anhydrous, 99.5\%) prepared in a planetary mixer (Thinky ARV-310), using a copper current collector foil (MTI, $12 \mu \mathrm{m})$. All solid components were filled into the mixer at once, adding NMP step-by-step ( 3 steps) and mixed at $2000 \mathrm{rpm}$ for $2 \mathrm{~min}$ per mixing step. The slurry was additionally mixed for 2 more min at $2000 \mathrm{rpm}$ just before the coating process. Coatings were dried in a self-built drying oven at $50^{\circ} \mathrm{C}$ under air for at least 12 hours. The scanning electron micrograph (SEM) of the electrode is shown in Figure 5. From the uncompressed electrode sheets, circular discs of various sizes were punched using an electrode punch (Hohsen Corp. OSAKA, Japan). $15 \mathrm{~mm}$ diameter glass fiber separator disks (binderfree glass microfiber 691 , thickness $200 \mu \mathrm{m}$, >90\% porosity, from VWR) were punched using a manual punching tool.

The determination of the porosity was done by measuring weight (Mettler Toledo, XP6, $\pm 1 \mu \mathrm{g}$ accuracy) and thickness (Mitutoyo Corporation, Tokyo, Japan, $\pm 2 \mu \mathrm{m}$ accuracy) of the electrodes. The bulk density of MCMB is assumed to be $2.2 \mathrm{~g} / \mathrm{cm}^{3}$. The absolute error of the electrode thickness measurement (without current collector) is $\pm 2 \mu \mathrm{m}$ (corresponding to $\pm 2 \%$ for electrode thicknesses ranging from $107-123 \mu \mathrm{m}$ ) and the absolute error of the weight measurement is estimated to be $\pm 0.1 \mathrm{mg} / \mathrm{cm}^{2}$. The electrodes were thus calculated to have a porosity of $48-50 \%$, a thickness (including the current collector) of $107-123 \mu \mathrm{m}$, a loading of $\sim 11.7-13.6 \mathrm{mg}_{\text {graphite }} / \mathrm{cm}^{2}$, and an areal capacity of $\sim 4.7 \mathrm{mAh} / \mathrm{cm}^{2}$ (calculated based on a theoretical capacity of $372 \mathrm{mAh} / \mathrm{g})$.

A 1:1 (w:w) mixture of ethylene carbonate (EC, Sigma Aldrich, anhydrous, 99\%) and diethyl carbonate (DEC, Sigma Aldrich, anhydrous, $>99 \%$ ) was used as a solvent for the self-prepared electrolytes containing various concentrations of tetrabutylammonium perchlorate $\left(\mathrm{TBAClO}_{4}\right.$, Sigma Aldrich $\left.\geq 99.0 \%\right)$ as conducting but nonintercalating salt. A turn-key conductivity sensor (LF 1100+, SI Analytics, with custom-made ground glass fitting) with a built-in temperature sensor was used to measure the conductivity of the electrolytes at $25^{\circ} \mathrm{C}$. The range of electrolyte conductivities used in this work varies between $2.65-7.10 \mathrm{mS} / \mathrm{cm}$ (corresponding to ca. $0.11 \mathrm{M}$ to $1 \mathrm{M}$ $\mathrm{TBAClO}_{4}$ in EC:DEC 1:1 (w:w), respectively).

Preparation of coin cells.-Figure 6 schematically shows the preparation steps involved in assembling the 2032-type coin cells (Hohsen Corp. OSAKA, Japan). First, $20 \mu \mathrm{L}$ of the electrolyte are placed onto the spacer disk (thickness $1 \mathrm{~mm}$ ) resting inside the coin 


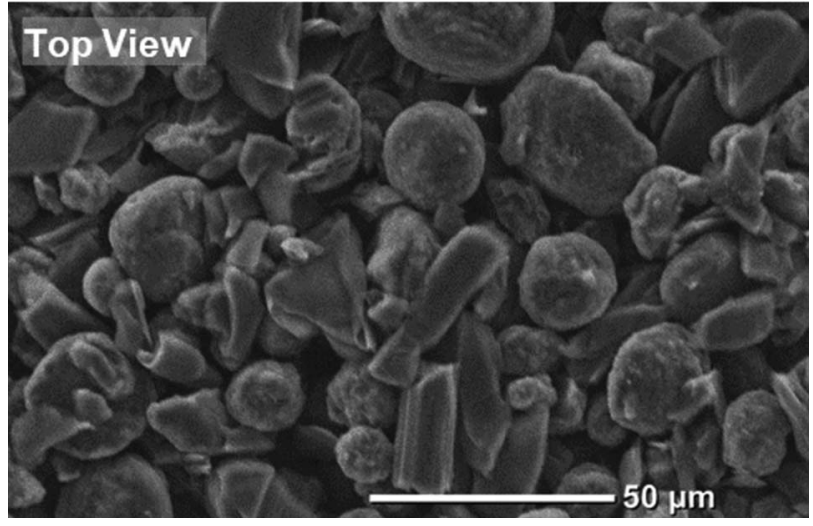

Figure 5. Scanning electron micrograph top-view of the electrode sheet (porosity of $48-50 \%$ ) prepared with $97 \%$ wt MCMB active material (MTI CORP, D50 $18 \mu \mathrm{m}$ ) and 3\% $\%_{\mathrm{wt}}$ PVDF binder (Kureha, KF1100).

cell casing top. The small amount of electrolyte $(20 \mu \mathrm{L})$ helps to more reliably locate the electrode disk (coating side facing down toward the spacer disk) at the center of the spacer disk. A glass fiber separator (diameter $15 \mathrm{~mm}$ ) is placed on top of the electrode (current collector side) and wetted with electrolyte $(40 \mu \mathrm{L})$, so that the perimeter of the electrode placed below becomes visible through the separator. This way, the second electrode which in the next step is being placed onto the separator (with the coating side facing up) can be aligned reliably with the first electrode. Subsequently, an additional $60 \mu \mathrm{L}$ of electrolyte are added to assure proper wetting of both electrode disks and the separator. Then another $1 \mathrm{~mm}$ thick spacer disk is placed on top of the second electrode disk. Finally, a spring is placed on top of the spacer disk and the cell is closed with the coin cell casing bottom. The cell is crimped using a 2032 crimping tool (Hohsen Corp. OSAKA, Japan). The total thickness of spacer disks in the coin cells is $2 \mathrm{~mm}$ unless stated otherwise. An argon filled and temperature controlled glove box (MBraun, $25^{\circ} \mathrm{C} \pm 1{ }^{\circ} \mathrm{C}$, oxygen and water content $<0.1$ ppm, Ar 5.0, Westfalen, 99.999\% vol.) was used to assemble the coin cells. It should be noted, however, that a glove box is not required to assemble the coin cells with the non-intercalating electrolyte for the here described tortuosity measurements; it was only used because the crimping tool was located there.

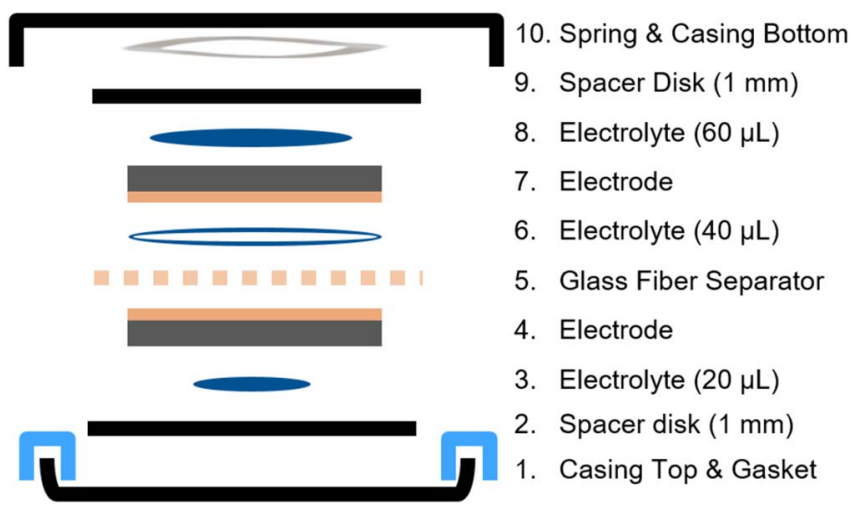

Figure 6. Sequence of steps detailing the process of assembling the 2032-type coin cells for the in-plane tortuosity measurement using two nominally identical electrode disks. In this design, the transport is predominantly in the in-plane direction of the electrode (gray bars), since the through-plane direction is blocked by the current collector (orange bars) and the spacer disks (black lines). A total of $120 \mu \mathrm{L}$ of electrolyte and of two $1 \mathrm{~mm}$ thick of spacer disks are used in all coin cells unless otherwise stated. Note that the 2032-type coin cell design allows the electrode disk diameter to be as big as $15 \mathrm{~mm}$.
EIS and cell testing procedures.-Electrochemical impedance spectra were measured around open circuit voltage (Biologic VMP3 potentiostat/galvanostat). A frequency range of $200 \mathrm{kHz}$ to $5 \mathrm{mHz}$ is used with a $10 \mathrm{mV}$ perturbation for measurements of the ionic resistance of the electrodes. All the measurements are obtained at least 48 hours after the assembly of the cells in order to ensure proper wetting, whereby the cells were allowed to equilibrate at $25^{\circ} \mathrm{C}$ for at least 2 hours prior to the measurements.

Additionally, two symmetric cells using a Swagelok T-cell setup were built to measure the through-plane tortuosity of the porous electrodes. The experimental setup and method to measure the throughplane tortuosity can be found in Landesfeind et al. ${ }^{1}$ The measured through-plane tortuosity for the here prepared electrode sheet was found to be $2.54 \pm 0.03$, where the error represents the standard deviation of two T-cell measurements. Throughout this paper, two coin cells were built for each configuration.

\section{Results and Discussion}

In this section, we first show representative impedance spectra for a base case along with the method to obtain the in-plane tortuosity. The previously mentioned radius invariance is presented next. Then, our proposed analysis method is validated by demonstrating that the obtained in-plane tortuosities are independent of electrode thickness and electrolyte conductivity, as would be expected based on the mathematical derivation presented in the Theory section. Finally, the robustness of the method with respect to electrode alignment imperfections is shown by intentionally misaligning the two electrode disks.

Experimental quantification of the in-plane tortuosity.-Two coin cells with $11 \mathrm{~mm}$ diameter disks (referred to as ' $\phi 11$ ' cells) were built using the procedure described in the Experimental setup section. The Nyquist plots of the impedance spectra are shown in Figure 7. While Figure 7a shows the full Nyquist plot, Figure 7b shows the zoomed-in view into the mid-and high-frequency region. The experimentally obtained Nyquist plots are very similar compared to the theoretically derived plots for pure capacitance (see Figure 2a), with the here obvious difference that the low-frequency tail of the Nyquist plot is not perfectly vertical (see Figure 7). As already mentioned above, this is related to the fact that the capacitance in porous electrodes is not perfectly described by a simple capacitor, but that it shows a constant-phase element behavior ${ }^{1}$ (the various factors leading to this behavior are discussed in the literature) ${ }^{32-34}$ By extrapolating the low-frequency tail in the Nyquist plot to the real axis and subtracting from the thus found value of the high-frequency intercept, the corrected low-frequency intercept $(L)$ can be obtained, from which the in-plane tortuosity can be calculated using Eq. 17. Note that the high frequency intercept consist of setup resistances as well as the electrolyte resistance between the perimeters of both the electrode disks. A homemade Matlab script is used to obtain the low-frequency intercept semi-automatically. The portion of the Nyquist plot to be used for linear extrapolation for the low-frequency intercept was determined by eye in all cases. For the two coin cells (' $\phi 11$ ') shown in Figure 7 , the in-plane tortuosity is found to be $1.65 \pm 0.01$, where the error represents the standard deviation of two coin cells. The experimental error in building each of the ' $\phi 11$ ' coin cells is $\pm 2 \%$ (the propagated error due to the measurement uncertainty of the electrode thickness and weight, of the electrolyte conductivity, and of the extrapolation for the low-frequency intercept). Table II lists all the parameters and measurements used to calculate the in-plane tortuosity along with the experimental errors.

Radius invariance of the electrode resistance.-As discussed in the Theory section, the resistance of the two electrodes measured in the configuration shown in Figure 1 should be independent of the disk diameter (see Figure 2a and Eq. 16). To validate that our theoretical derivation indeed reflects experimental measurement, two coin cells with electrode disk diameters of $14 \mathrm{~mm}$ were made (referred to as ' $\phi 14$ ' cells). The Nyquist plots of these ' $\phi 14$ ' coin cells are shown 
Table II. Details of the individual cells and electrodes together with the experimentally obtained in-plane tortuosity data $\tau_{i-p}$. All tested cells contain one glass fiber separator (uncompressed thickness $200 \mu \mathrm{m}, 15 \mathrm{~mm}$ diameter) and all graphite electrodes are uncalendered/uncompressed and have a diameter of $11 \mathrm{~mm}$, except for the ' $\phi 14$ ' design which uses $14 \mathrm{~mm}$ diameter disks. The cells referred to as 'thicker' use a lower spacer thickness ( $1.5 \mathrm{~mm}$ compared to $2 \mathrm{~mm}$ total spacer thickness) and more electrolyte ( $135 \mu \mathrm{L}$ instead of $120 \mu \mathrm{L})$ compared to all other cell in order to accommodate the doubled-up 'thicker' electrodes for this configuration (see Figure 8). The porosity variation of the coating is between 0.48-0.49 and the thickness of individual electrodes (w/o current collector) varies between 107-123 $\mu \mathrm{m}$ (loadings of $\sim 11.7-13.6 \mathrm{mg}_{\text {graphite }} / \mathrm{cm}^{2}$ ). The porosity and electrode thickness reported here for each coin cell are the average of the two electrode disks used to make the cell.

\begin{tabular}{|c|c|c|c|c|c|c|c|c|c|}
\hline Design & Cell \# & Disk Diameter mm & Spacer Thickness* mm & Volume of Electrolyte $\mu \mathrm{L}$ & $\varepsilon$ & $d \mu \mathrm{m}$ & $\kappa \mathrm{mS} / \mathrm{cm}$ & $L^{\dagger} \mathrm{k} \Omega$ & $\tau_{\mathrm{i}-\mathrm{p}}^{\ddagger}$ \\
\hline \multirow[t]{2}{*}{$\phi 11$} & $\# 1$ & 11 & $1+1$ & 120 & 49.1 & 114 & 7.10 & 3.28 & $1.64 \pm 2 \%$ \\
\hline & \#2 & & & & 49.1 & 114 & 7.10 & 3.32 & $1.66 \pm 2 \%$ \\
\hline \multirow[t]{2}{*}{$\phi 14$} & \#1 & 14 & $1+1$ & 120 & 48.2 & 113 & 7.10 & 3.54 & $1.72 \pm 3 \%$ \\
\hline & $\# 2$ & & & & 48.0 & 120 & 6.86 & 3.50 & $1.74 \pm 2 \%$ \\
\hline \multirow[t]{2}{*}{ 'thicker' } & $\# 1$ & 11 & $1+0.5$ & 135 & 48.2 & 225 & 7.10 & 1.90 & $1.84 \pm 2 \%$ \\
\hline & \#2 & & & & 48.4 & 226 & 7.10 & 1.95 & $1.91 \pm 2 \%$ \\
\hline \multirow[t]{2}{*}{ 'low-к' } & $\# 1$ & 11 & $1+1$ & 120 & 49.0 & 117 & 2.65 & 7.89 & $1.51 \pm 2 \%$ \\
\hline & $\# 2$ & & & & 49.1 & 118 & 2.65 & 7.68 & $1.48 \pm 2 \%$ \\
\hline \multirow[t]{2}{*}{ 'misaligned' } & $\# 1$ & 11 & $1+1$ & 120 & 49.9 & 107 & 6.86 & 3.94 & $1.81 \pm 3 \%$ \\
\hline & $\# 2$ & & & & 49.2 & 112 & 6.86 & 3.75 & $1.78 \pm 3 \%$ \\
\hline
\end{tabular}

\footnotetext{
* Spacer thickness indicates the sum of two spacers.

${ }^{\dagger}$ Note that the value $L$ represents the extrapolated low-frequency intercept corrected for the high-frequency intercept (the high-frequency intercept is $\sim 3-9$ $\Omega$ for the ' $\phi 11$ ', ' $\phi 14$ ' and 'low- $\kappa$ ' cells, 12-30 $\Omega$ for thicker cells and $\sim 5-113 \Omega$ for the 'misaligned' cells).

$\ddagger$ The error in individual values of the in-plane tortuosity is based on the propagated error due to the measurement uncertainty of the electrode thickness and weight, of the electrolyte conductivity, and of the extrapolation for the low-frequency intercept.
}

in Figure 8, along with a comparison to the impedance data for the ' $\phi 11$ ' coin cells shown in Figure 5. Since the electrode thicknesses, porosities, and loadings as well as the coin cell assembly/testing specifications are essentially identical, the analytical solution shown in Figure 2a would predict that the Nyquist plots for these electrodes which differ only in diameter should be identical within the margin of experimental error. As can be seen in Figure 8, this is indeed the case. Table II shows the electrode and coin cell specifications together with the values of the in-plane tortuosity obtained from the Nyquist plot analysis (determination of $L$ ) of these two sets of cells with 11 and $14 \mathrm{~mm}$ diameter in combination with Eq. 17. The $L$ and $\tau_{\mathrm{i}-\mathrm{p}}$ values for these cells are essentially identical, validating the theoretically predicted invariance of the disk resistance and the obtained in-plane tortuosity values with respect to the disk diameter.

Additional validation.- Two possible ways to buttress the validity of the proposed in-plane tortuosity measurement method are to test it on different electrode thicknesses (referred to as 'thicker' cells) and with different electrolyte conductivities (referred to as 'low-א' cells).

Doubling electrode thickness ('thicker' cells).-The thickness of the electrodes was doubled by putting two electrode disks $(11 \mathrm{~mm}$ diameter) together as shown in Figure 9. In this case, the total spacer thickness must be reduced (to $1.5 \mathrm{~mm}$ from the standard configuration of $2 \mathrm{~mm}$ ) to compensate for the thicker electrodes to avoid too high compression in the coin cell setup. Also, to compensate for the increase in void volume for having two additional electrode disks (combined additional volume of $\sim 11 \mu \mathrm{L}$, based on two additional $11 \mathrm{~mm}$ diameter electrodes with a thickness $113 \mu \mathrm{m}$ and a porosity of $\sim 0.48$ ), $135 \mu \mathrm{L}$ of the electrolyte was used as opposed to $120 \mu \mathrm{L}$ in the standard configuration. Having similar values of all other parameters (e.g. porosity, tortuosity and electrolyte conductivity), the value of $L$ (low-frequency intercept corrected for the high-frequency intercept) should be halved for the 'thicker' cells compared to the ' $\phi 11$ ' cells.

Figure 10 shows the impedance spectra of the 'thicker' cells along with the ' $\phi 11$ ' cell data from Figure 5 for comparison. Table II shows the measured properties of the electrodes (porosity $\varepsilon$, and thickness $d$ ) and the electrolyte (bulk conductivity $\kappa$ ) as well as of the low-frequency resistance $L$ (corrected for the high-frequency resistance) which are needed for the calculation of the in-plane tortuosity $\tau_{i-p}$ of the 'thicker' cells. As shown in Table II, the in-plane tortuosi- ties obtained from the measurements with the doubled-up electrodes ('thicker') are $\sim 10-15 \%$ larger than those measured for the singleelectrode measurements (' $\phi 11$ ' and ' $\phi 14$ '). The increased value of tortuosity may be due to the fact that the calculation of the in-plane tortuosity is done based on the sum of the individually measured single electrode thicknesses rather than the thickness of the doubled-up combined disks, which might differ due to the electrodes being not calendered. It is also to be noted that 'thicker' cell setup consists of four electrode disks, where alignment is difficult to achieve compared to the ' $\phi 11$ ' and the ' $\phi 14$ ' cell setups (two disks per cell).

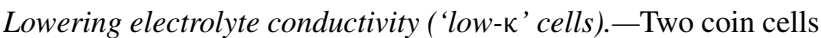
with $11 \mathrm{~mm}$ diameter electrodes and with lower conductivity electrolyte $(\kappa=2.65 \mathrm{mS} / \mathrm{cm})$ were built as an additional validation to the proposed method. Figure 10 shows the impedance spectra of 'low- $\kappa$ ' cells along with the 'thicker' and the ' $\phi 11$ ' cells. Table II shows the electrode and electrolyte specifications along with the values of the low-frequency intercept (corrected for the high-frequency resistance) and the in-plane tortuosity. The tortuosity values obtained from the 'low- $\kappa$ ' cell design agree reasonably well with the in-plane tortuosity obtained from the ' $\phi 11$ ' cell designs $(\sim 10 \%$ smaller compared to ' $\phi 11$ ' cells). In principle, the best setup for accurate in-plane tortuosity measurement is based on a low conductivity electrolyte (see Refs. 1 and 26) that still allows for linear extrapolation at frequencies smaller than the characteristic frequency. For practical reasons (measurement time, temperature fluctuations in the climate-chamber) the lowest measurement frequency is limited to the $\mathrm{mHz}$ range. Hence the electrolyte conductivity cannot be reduced too much as the low frequency constant phase element will not be observable in the frequency range of the measurement anymore.

We apply the analysis discussed in the Theory section with regards to the turning frequency on the 'low- $\kappa$ ' cells. The low frequency intercept value for 'low- $\kappa$ ' cells is $\sim 7.8 \mathrm{k} \Omega$ (by eye extrapolation in the Nyquist plot). The approximate turning frequency where the imaginary part of impedance is close to $7.8 \mathrm{k} \Omega$ is $\sim 50 \mathrm{mHz}$. The characteristic frequency is at least an order of magnitude lower than the turning frequency and is approximately $4 \mathrm{mHz}$ (see Figure 3). Note that the minimum frequency in all our experiments is set to $5 \mathrm{mHz}$. For the 'low- $\kappa$ ' cells, the choice of $5 \mathrm{mHz}$ as the minimum frequency explains the underprediction of the low frequency intercept (and thus the in-plane tortuoisty) compared to the other cell setups to some extent. 

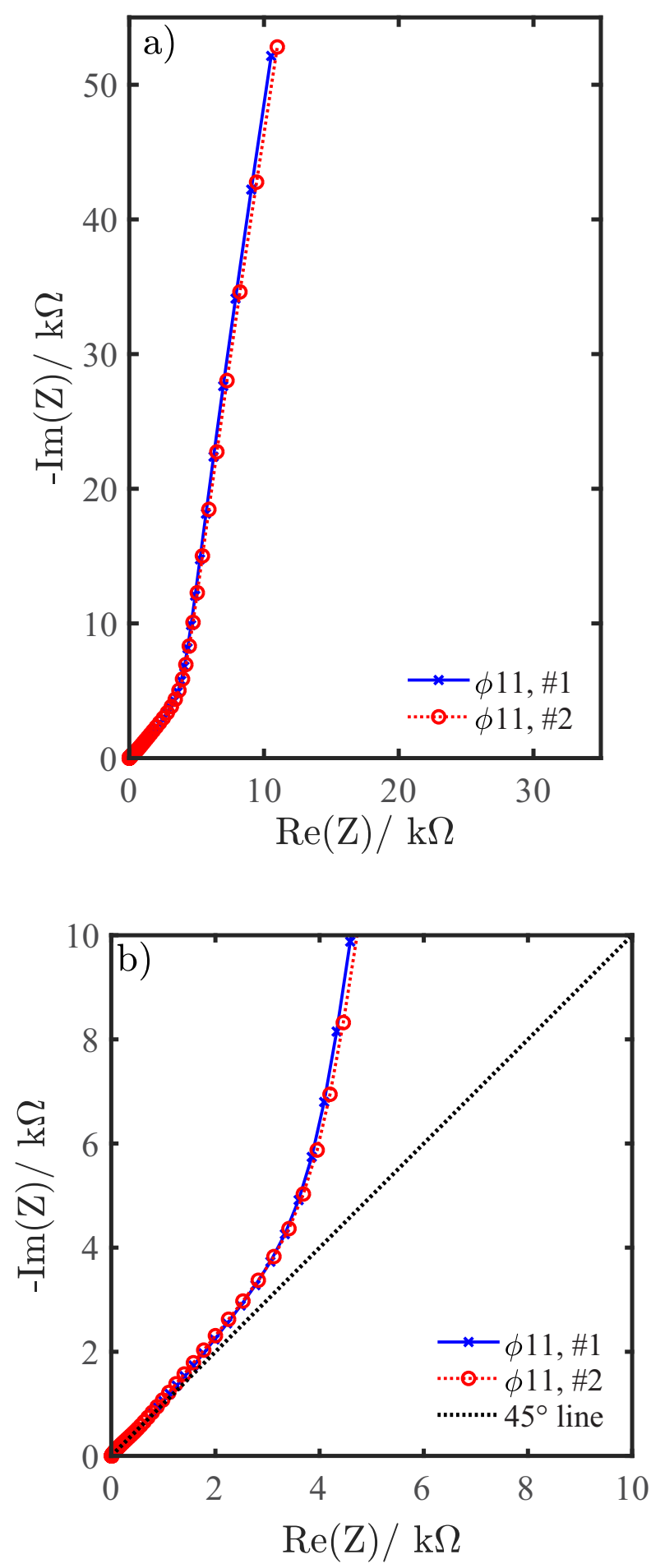

Figure 7. Nyquist plots of two coin cells with $11 \mathrm{~mm}$ diameter electrodes (referred to as ' $\phi 11$ ') obtained from EIS measurements (perturbation of $10 \mathrm{mV}$, recorded at $25^{\circ} \mathrm{C}$ after $48 \mathrm{~h}$ ). Figure 7 a shows the impedance spectrum over the full frequency range $(200 \mathrm{kHz}$ to $5 \mathrm{mHz})$ and Figure $7 \mathrm{~b}$ is a zoomed-in view ( $200 \mathrm{kHz}$ to $\sim 31 \mathrm{mHz}$ ) with the black dotted line of slope 1 (' $45^{\circ}$ line') for reference. Electrode specifications: uncalendered/uncompressed electrode disks $\left(97 \%_{\mathrm{wt}} \mathrm{MCMB}, 3 \%_{\mathrm{wt}} \mathrm{PVDF}\right)$ with an average electrode thickness of $114 \mu \mathrm{m}$ (without current collector), a loading of $\sim 12.6 \mathrm{mg}_{\text {graphite }} / \mathrm{cm}^{2}$, and a porosity of $\sim 49 \%$. Coin cell specifications: two $1 \mathrm{~mm}$ thick spacers, one glass fiber separator (uncompressed thickness $200 \mu \mathrm{m}$, porosity > 90\%), and a total electrolyte volume of $120 \mu \mathrm{L}$ with an electrolyte conductivity of $7.10 \mathrm{mS} / \mathrm{cm}$ at $25^{\circ} \mathrm{C}\left(\sim 1 \mathrm{M} \mathrm{TBAClO}_{4}\right.$ in EC:DEC 1:1 (w:w)). Extrapolation of the linear region of the low-frequency range of the Nyquist plot (corrected by the high-frequency intercept) gives $L \approx 3.3 \mathrm{k} \Omega$, which is then used to calculate the in-plane tortuosity (see Eq. 17 and Table II).

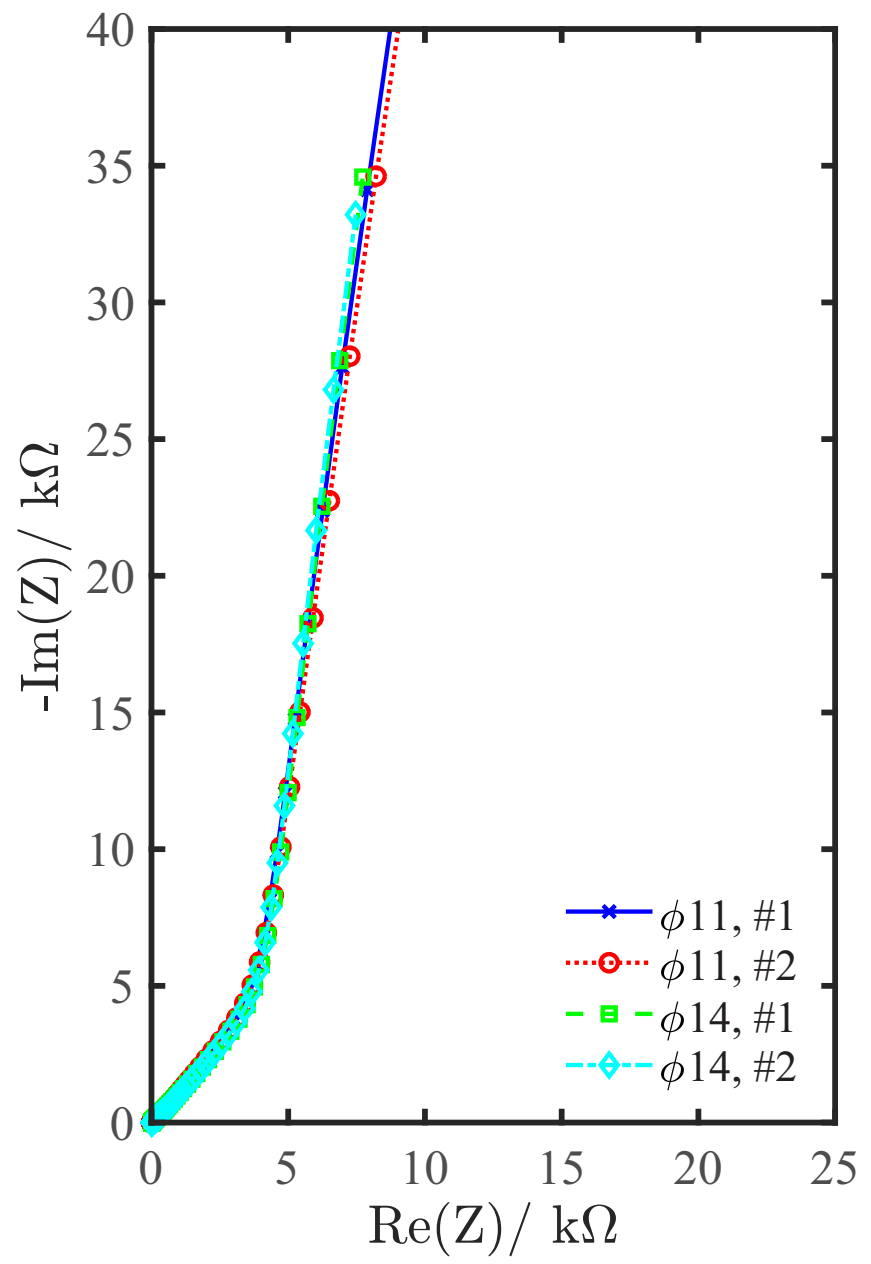

Figure 8. Nyquist plots based on essentially identical electrodes punched out to either $14 \mathrm{~mm}$ diameter (' $\phi 14$ ' coin cells) or to $11 \mathrm{~mm}$ (data from ' $\phi 11$ ' cells shown in Figure 7a), based on EIS measurements (perturbation of $10 \mathrm{mV}$, $200 \mathrm{kHz}$ to $5 \mathrm{mHz}$ at $25^{\circ} \mathrm{C}$, recorded after $48 \mathrm{~h}$ ). Electrode specifications: uncalendered/uncompressed electrode disks (97\% $\%_{\mathrm{wt}}$ MCMB, 3\% $\%_{\mathrm{wt}}$ PVDF) with an average electrode thickness of 113-120 $\mu \mathrm{m}$ (without current collector), a loading of $\sim 12.7-13.5 \mathrm{mg}_{\text {graphite }} / \mathrm{cm}^{2}$, and a porosity of $\sim 48-49 \%$. Coin cell specifications: two $1 \mathrm{~mm}$ thick spacers, one glass fiber separator (uncompressed thickness $200 \mu \mathrm{m}$, porosity $>90 \%$ ), and a total electrolyte volume of $120 \mu \mathrm{L}$ with an electrolyte conductivity of $6.86-7.10 \mathrm{mS} / \mathrm{cm}$ at $25^{\circ} \mathrm{C}(\sim 1 \mathrm{mM}$ $\mathrm{TBAClO}_{4}$ in EC:DEC 1:1 (w:w)). See Table II for the details of the individual cell parameters and measurements.

Error tolerance.-In this subsection, the error tolerance of the proposed method is shown by building two coin cells with intentionally misaligned disks (referred to as 'misaligned' cells). The inset of Figure 11 shows the significant misalignment of one of the two "misaligned' cells. The image was taken just before the crimping process of the coin cell. Figure 11 also shows the Nyquist plots of the two misaligned cells along with the ' $\phi 11$ ' data from well-aligned electrodes as a reference case. The tortuosity obtained for the intentionally misaligned cells was measured to be only $10 \%$ higher compared to the tortuosity obtained from the ' $\phi 11$ ' cell setup (see Table II), which demonstrates the reasonably good robustness of the proposed methodology toward experimental inaccuracy.

Discussion.-The five different cell setups-varying disk diameters, electrode thicknesses, electrolyte conductivities, and alignment-were investigated in this work and the results are summarized in Table II. Although, the values obtained from all the cell setups are within $\sim 15 \%$ of the ' $\phi 11$ ' cells, it begs a very important question, 'what value of $\tau_{i-p}$ is most reliable?' Here, we try to answer 


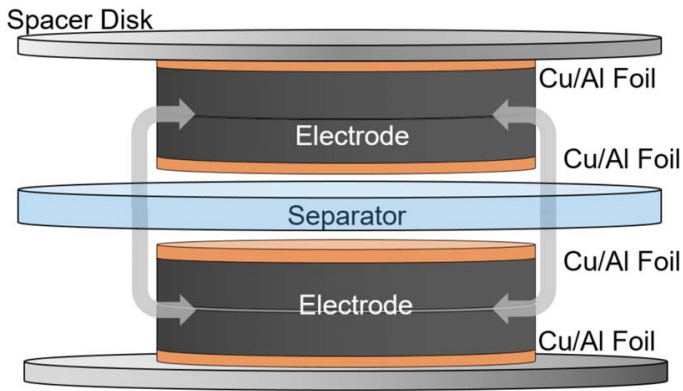

Spacer Disk

Figure 9. Schematic of the 'thicker' cell design. Two nominally identical electrode disks are used on each side of the symmetric cell. Compared to the other cell design (see Figure 1), the 'thicker' cell design has a total spacer disk thickness of $1.5 \mathrm{~mm}$ (rather than to $2 \mathrm{~mm}$ ) in order to accommodate the higher thickness of the electrode stack. The amount of electrolyte used was also increased from $120 \mu \mathrm{L}$ to $135 \mu \mathrm{L}$. All other coin cell design parameters (glass fiber separator, disk radius, electrolyte conductivity, etc.) are identical to the ' $\phi 11$ ' cell design (Figure 1).

this question only qualitatively using the analysis performed in the Theory section.

As discussed before, for linear extrapolation, the access to low-frequency measurements (without much experimental error) compared to the characteristic frequency is desirable for better estimation of the low-frequency intercept. Ignoring other sources of errors that might be present in the low-frequency range, and arguing purely from the TLM analysis, the values obtained from the ' $\phi 11$ ' and 'thicker' cells should be closer to the 'true' values of the in-plane tortuosity. It has to be noted that a possible misalignment in the 'thicker' cells, due to the presence of four disks as opposed to two disks, may result in higher low-frequency intercepts and make the method more error prone experimentally. Also, it can be seen from the Theory section that the low conductivity cell setup ('low- $\kappa$ ') should have the highest under-prediction of the resistance (hence tortuosity), which is indeed seen from the 'low-к' cells.

\section{Conclusions}

This paper presents a novel method and experimental coin-cell based setup to determine the in-plane tortuosity of a porous electrode using a non-intercalating electrolyte and the application of a blocking condition transmission line model. The proposed method yields a very elegant mathematical description of the in-plane tortuosity, which was shown to be invariant of the radius of the tested electrode and to be rather tolerant with regards to electrode misalignment which may result during cell assembly. The theoretical analysis performed with the constant phase element provides some guidelines with regards to the experimental design. The characteristic frequency is at least an order of magnitude smaller than the turning frequency (see Figure 3 ), hence to avoid high systematic underestimation of the 'true' lowfrequency intercept, the experimental design should allow access to the lower frequencies (lower than $f_{c}$ ) without having to go to less than $\sim 1 \mathrm{mHz}$ (measurements for less than $1 \mathrm{mHz}$ frequency range may be corrupted by temperature fluctuations, side reactions and require significantly more testing time). The method was validated by comparing the in-plane tortuosity values measured for uncalendered/uncompressed graphite electrodes $\left(97 \%_{\mathrm{wt}} \mathrm{MCMB}, 3 \%_{\mathrm{wt}} \mathrm{PVDF}\right.$, porosity $48 \%-50 \%$ ) using different experimental conditions (electrode thickness and electrolyte conductivity). The in-plane tortuosity value $\left(\tau_{\mathrm{i}-\mathrm{p}} \sim 1.6\right)$ is smaller than the through-plane tortuosity $\left(\tau_{t-p}=2.3\right)$. It should be noted that the previous determination (by Landesfeind et al.) ${ }^{1}$ of the through-plane tortuosity for graphite anodes $\left(\tau_{t-p} \approx 5\right)$ was done for electrodes made from flake-like graphite particles using a different preparation procedure as compared to the electrodes used in this work (spherical MCMB particles, see Figure 5). The in-plane tortuosity is close
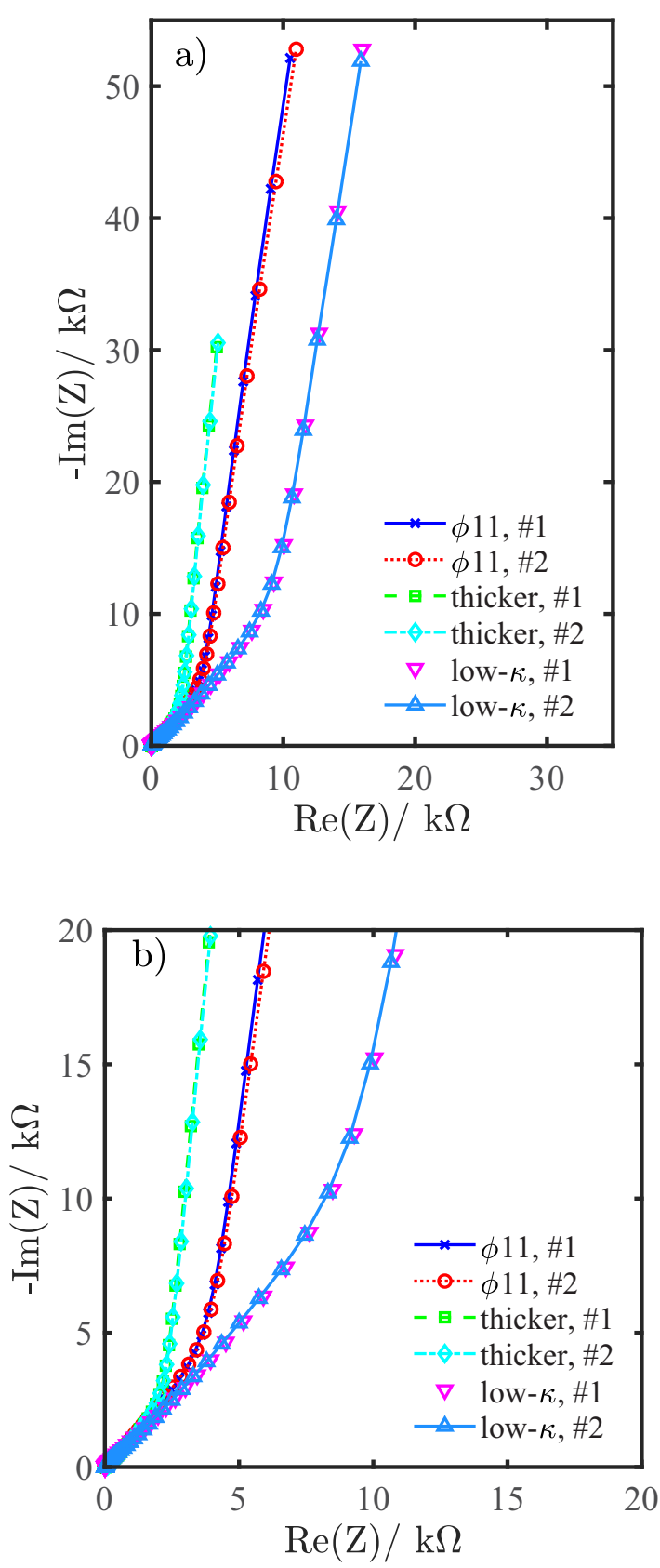

Figure 10. a. Impedance curves obtained for uncalendered/uncompressed graphite electrodes $\left(97 \%_{\mathrm{wt}} \mathrm{MCMB}, 3 \%_{\mathrm{wt}} \mathrm{PVDF}\right.$ ) in three different coin cell designs ('thicker', ' $\phi 11$ ', and 'low- $\kappa$ ') obtained from EIS measurements (perturbation of $10 \mathrm{mV}, 200 \mathrm{kHz}-5 \mathrm{mHz}$ at $25^{\circ} \mathrm{C}$, recorded after $48 \mathrm{~h}$ ). b. Zoomed-in view of the impedance plots. The 'thicker' cells have nominally double the electrode thickness compared to the ' $\phi 11$ ' cells; the 'low- $\kappa$ ' cells have an electrolyte conductivity of $2.65 \mathrm{mS} / \mathrm{cm}$ compared to $7.1 \mathrm{mS} / \mathrm{cm}$ for the ' $\phi 11$ ' cells ( $\sim 0.11 \mathrm{M}$ vs. $\sim 1 \mathrm{M} \mathrm{TBAClO}_{4}$ in EC:DEC 1:1 (w:w), respectively). Detailed cell and electrode specifications are given in Table II.

to the Bruggeman expression (see Figure 12), whereby the remaining difference between the experimental values obtained and the Bruggeman expression might be due to the presence of binder $(3 \%$ wt PVDF) or a deviation of the particles from spherical geometry. The work done by Landesfeind et al. has shown how various factors such as particle shape, binder content (or binder layer thickness) and porosity (compression) can affect the through-plane tortuosity. ${ }^{1,2}$ It will be interesting to see how such parameters affect the in-plane tortuosity and what govern the tortuosity anisotropy in battery electrodes, which is the focus of an ongoing study. 


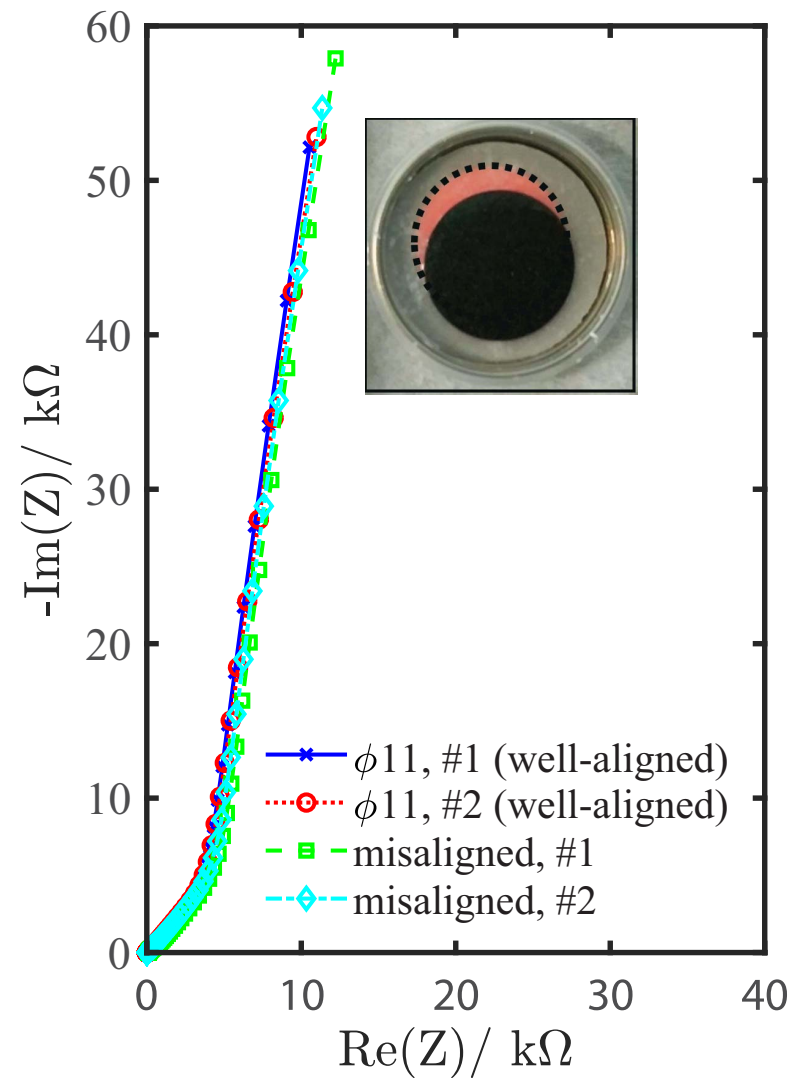

Figure 11. Impedance spectra of deliberately 'misaligned' graphite electrodes (11 mm diameter) along with the ' $\phi 11$ ' data (Figure 5) obtained from EIS measurements (perturbation of $10 \mathrm{mV}, 200 \mathrm{kHz}-5 \mathrm{mHz}$ at $25^{\circ} \mathrm{C}$, recorded after $48 \mathrm{~h}$ ). All other cell design parameters are similar as the ' $\phi 11$ ' coin cell design. The Inset shows the image of one of the 'misaligned' coin cells where considerable misalignment was maintained between the two disks. The black dotted outline is drawn on the bottom electrode to increase the clarity of the image, which was taken just before the crimping process. Detailed cell and electrode specifications are given in Table II.

The ability to measure the in-plane tortuosity of battery electrodes opens the door to analyze the effect of several parameters in the electrode preparation process. The combined use of in-plane and throughplane tortuosity can also shed light on various inhomogeneities that might be present in an electrode and on the effect of binder layer thickness, binder content, drying method, ink preparation routine, particle size distribution, particle shape and calendering. This method can also be used to determine the in-plane tortuosity of gas diffusion layers used in fuel cells, as will be shown in our future work.

\section{Acknowledgment}

We thank the BMBF (Federal Ministry of Education and Research, Germany) for its financial support under the auspices of the ExZellTUM II project (grant number 03XP0081). B.S. thanks Robert Morasch, Tanja Zünd, Anna Freiberg, Manu Patel and Morten Wetjen for helpful discussions.

\section{ORCID}

Bharatkumar Suthar (D) https://orcid.org/0000-0002-8612-9483 Johannes Landesfeind (iD https://orcid.org/0000-0003-0333-2185

\section{References}

1. J. Landesfeind, J. Hattendorff, A. Ehrl, W. A. Wall, and H. A. Gasteiger, Journal of The Electrochemical Society, 163, A1373 (2016).

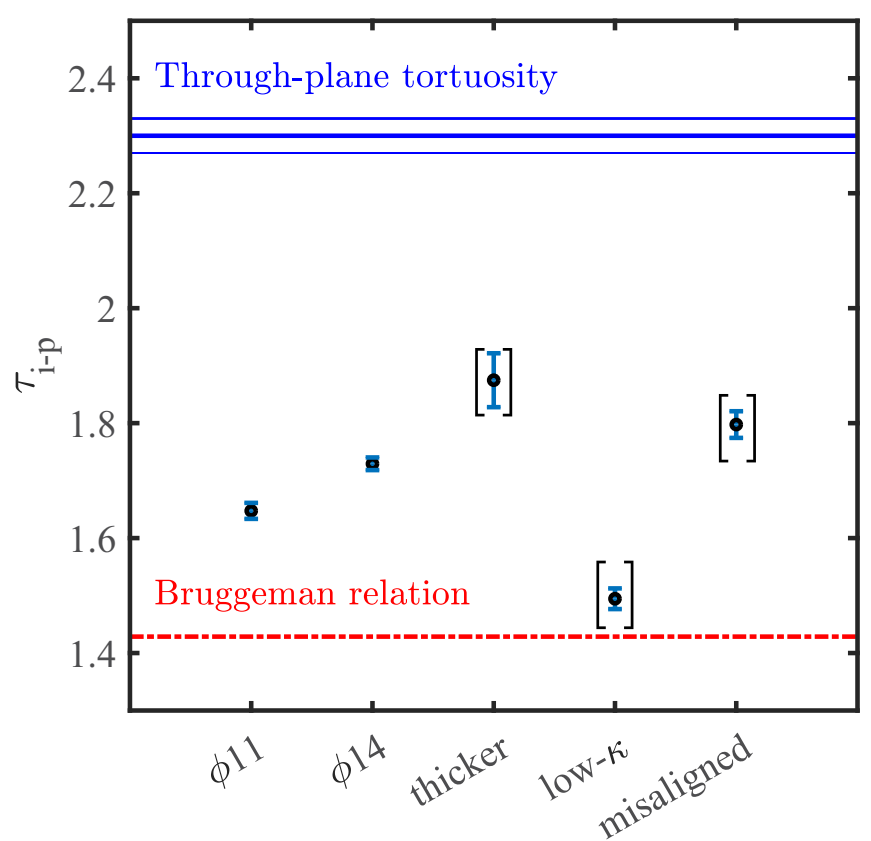

Figure 12. Summary of the measured in-plane tortuosity values of noncalendered/noncompressed graphite electrodes $\left(97 \%_{\mathrm{wt}}\right.$ MCMB and 3\% $\%_{\mathrm{wt}}$ PVDF, with $48-50 \%$ porosity) from different cell setups. The error bars represent the standard deviation of the mean values of two independent repeat measurements (see Table II, the error in individual values of the in-plane tortuosity-which is $2-3 \%$, stemming from thickness and conductivity errors-is not incorporated in the error bars). The 'thicker' cells and the 'misaligned' cell setups are prone to measurement errors (alignment and thickness determination issues), and the 'low- $\kappa$ ' cells are prone to larger extrapolation underprediction compared to high conductivity electrolyte setups, hence indicated in brackets. Also shown for comparison are the tortuosity derived from the Bruggeman relation for spherical particles $\left(\tau_{i-p}=1 / \sqrt{\varepsilon}\right.$, with $\varepsilon \approx 0.49$, red dash-dot line) and the through-plane tortuosity of the electrode (solid blue line; the shaded blue region represents the error bar from two T-cells). For details on the measurement setup/procedure and the electrode specifications see Table II.

2. J. Landesfeind, A. Eldiven, and H. A. Gasteiger, Journal of The Electrochemical Society, 165, A1122 (2018)

3. M. M. Forouzan, C.-W. Chao, D. Bustamante, B. A. Mazzeo, and D. R. Wheeler, Journal of Power Sources, 312, 172 (2016).

4. M. Stein, A. Mistry, and P. P. Mukherjee, Journal of The Electrochemical Society, 164, A1616 (2017).

5. D. Pritzl, J. Landesfeind, S. Solchenbach, and H. A. Gasteiger, submitted.

6. J. W. Long, B. Dunn, D. R. Rolison, and H. S. White, Chemical Reviews, 104, 4463 (2004).

7. J. H. Pikul, H. Gang Zhang, J. Cho, P. V. Braun, and W. P. King, Nature Communications, 4, 1732 (2013)

8. P. Smyrek, J. Pröll, H. J. Seifert, and W. Pfleging, Journal of The Electrochemical Society, 163, A19 (2016)

9. B. Ketterer, H. Vasilchina, K. Seemann, S. Ulrich, H. Besser, W. Pfleging, T. Kaiser, and C. Adelhelm, International Journal of Materials Research, 99, 1171 (2008).

10. E. Mottay, X. Liu, H. Zhang, E. Mazur, R. Sanatinia, and W. Pfleging, MRS Bulletin, 41, 984 (2016).

11. J. B. Habedank, J. Endres, P. Schmitz, H. P. Huber, and M. F. Zaeh, submitted.

12. M. Müller, L. Pfaffmann, S. Jaiser, M. Baunach, V. Trouillet, F. Scheiba, P. Scharfer, W. Schabel, and W. Bauer, Journal of Power Sources, 340, 1 (2017).

13. J. C. Burns, A. Kassam, N. N. Sinha, L. E. Downie, L. Solnickova, B. M. Way, and J. R. Dahn, Journal of The Electrochemical Society, 160, A1451 (2013).

14. M. Ebner, D.-W. Chung, R. E. García, and V. Wood, Advanced Energy Materials, 4 (2014).

15. C. Ding-Wen, E. Martin, R. E. David, W. Vanessa, and R. E. García, Modelling and Simulation in Materials Science and Engineering, 21, 074009 (2013).

16. S. J. Cooper, D. S. Eastwood, J. Gelb, G. Damblanc, D. J. L. Brett, R. S. Bradley, P. J. Withers, P. D. Lee, A. J. Marquis, N. P. Brandon, and P. R. Shearing, Journal of Power Sources, 247, 1033 (2014).

17. M. Ender, J. Joos, T. Carraro, and E. Ivers-Tiffée, Journal of The Electrochemical Society, 159, A972 (2012).

18. M. Ebner and V. Wood, Journal of The Electrochemical Society, 162, A3064 (2015).

19. I. V. Thorat, D. E. Stephenson, N. A. Zacharias, K. Zaghib, J. N. Harb, and D. R. Wheeler, Journal of Power Sources, 188, 592 (2009). 
20. N. Ogihara, S. Kawauchi, C. Okuda, Y. Itou, Y. Takeuchi, and Y. Ukyo, Journal of The Electrochemical Society, 159, A1034 (2012).

21. L. Holzer, D. Wiedenmann, B. Münch, L. Keller, M. Prestat, P. Gasser, I. Robertson, and B. Grobéty, Journal of Materials Science, 48, 2934 (2013).

22. D. R. Baker, D. A. Caulk, K. C. Neyerlin, and M. W. Murphy, Journal of The Electrochemical Society, 156, B991 (2009).

23. M. F. Mathias, J. Roth, J. Fleming, and W. Lehnert, Diffusion media materials and characterization, in Handbook of Fuel Cells, W. Vielstich, A. Lamm, H. A. Gasteiger, and H. Yokokawa Editors, John Wiley \& Sons, Ltd (2010).

24. R. Rashapov, F. Imami, and J. T. Gostick, International Journal of Heat and Mass Transfer, 85, 367 (2015)

25. D. Kramer, S. A. Freunberger, R. Flückiger, I. A. Schneider, A. Wokaun, F. N. Büchi, and G. G. Scherer, Journal of Electroanalytical Chemistry, 612, 63 (2008).

26. J. Landesfeind, M. Ebner, A. Eldiven, V. Wood, and H. A. Gasteiger, Journal of The Electrochemical Society, 165, A469 (2018).
27. V. F. Lvovich, Impedance spectroscopy: applications to electrochemical and dielectric phenomena, John Wiley \& Sons (2012).

28. J. Newman and K. E. Thomas-Alyea, Electrochemical systems, John Wiley \& Sons (2012).

29. D. D. Macdonald, Electrochimica Acta, 51, 1376 (2006)

30. J. Landesfeind, D. Pritzl, and H. A. Gasteiger, Journal of The Electrochemical Society, 164, A1773 (2017).

31. A. Lasia, Electrochemical Impedance Spectroscopy and its Applications, Springer New York (2014).

32. G. J. Brug, A. L. G. van den Eeden, M. Sluyters-Rehbach, and J. H. Sluyters, Journal of Electroanalytical Chemistry and Interfacial Electrochemistry, 176, 275 (1984).

33. A. Lasia, Journal of Electroanalytical Chemistry, 397, 27 (1995).

34. J.-B. Jorcin, M. E. Orazem, N. Pébère, and B. Tribollet, Electrochimica Acta, 51 1473 (2006). 